\title{
Influence of some operational variables on the radial keratotomy operation
}

Biomechanical and Medical Information Institute, Beijing Polytechnic University, Beijing, PRC

J-Q Wang

Y-J Zeng

Department of Ophthalmology, Beijing Friendship Hospital, Capital Medical University, Beijing, PRC $\mathrm{X}-\mathrm{Y} \mathrm{Li}$

Correspondence to: Dr Wang

yjzeng@bjpu.edu.cn

Accepted for publication 13 January 2000

Jia-Quan Wang, Yan-Jun Zeng, X-Y Li

\begin{abstract}
Aims-To assess the contribution of the four major operational variables in the radial keratotomy operation (RK) to the correction of myopic eyes. To study the deformation of the cornea after the operation and provide some valuable references for clinical practice.

Methods-The expression of the correction ratio has been deduced, which can be directly represent as the deformation ratio of the corneal radius after the operation. This allows for the numerical simulation of the RK operation. On the basis of the known biomechanical property of the cornea, by means of the finite element method, a series of computerised geometric and biomechanical models for the simulation of refractive surgery have been established. Some operational variables in the RK operation were analysed, and their influence on the operational outcome has been computed and systematically analysed.

Results and conclusion-All of the four variables have a positive effect on the operational outcome. The greater the four variables are the more correction effects of myopia can be obtained. Because the four variables are interinfluential, the operational variables can be optimised to get the best result in order to meet patients' differing requirements.
\end{abstract}

(Br f Ophthalmol 2000;84:651-653)

Radial keratotomy (RK) is a widely accepted surgical procedure to correct myopia. In the operation, sets of 4-12 evenly spaced radial incisions are made in the treated cornea, which extend from the boundary of a central optical clear zone of specified diameter to the outer periphery of the cornea. The intraocular pressure causes the weakened peripheral region of the cornea to bulge outward and flatten the central visual region by stretching it like a drum head. The central flattening reduces the refractive power of the anterior corneal surface, and the focal point is shifted posteriorly to the surface of the retina producing corneal deformation after the operation. The effectiveness of this procedure depends on the surgeon's ability to select surgical variables that lead to the desired reduction in the refractive power. In order to predict the effect of the operation many statistical models have been developed in carefully controlled medical studies. Russian scientists did this work many decades ago and gained a successful effect that can be utilised in the clinical operation. ${ }^{1}$ But statistical studies do not provide a complete understanding of corneal mechanics, preventing further research work. Therefore, a study of corneal biomechanics is the first step in the construction of biomechanical models for the myopic eye. The biomechanical properties of the cornea utilised here are provided by other researchers in the same laboratory, who have been doing this work for many years and have gained very satisfactory results. ${ }^{23}$ In this paper, computerised biomechanical models of the moderately myopic cornea for the simulation of refractive surgery were established, many of which were optimised with the help of ANsys finite element software.

\section{Methods}

According to ophthalmological refractive optics, the expression of refractive power $\mathrm{D}$ is

$$
D=\frac{n_{2}-n_{1}}{r}
$$

where $r$ is the curvature radius of the cornea, $\mathrm{n}_{2}$ and $\mathrm{n}_{1}$ are the refractive index of the cornea and the air respectively. ${ }^{4}$

The differential of refractive power $\mathrm{D}$ is

$$
d D=-\frac{n_{2}-n_{1}}{r^{2}} d r
$$

in small deformation condition,

$$
\Delta D=-\frac{\left(n_{2}-n_{1}\right) \Delta r}{r^{2}}
$$

which means the correction quantity of the refractive power at the specific change of the curvature radius $r$ of the operated cornea. The percentage correction ratio $\eta$ is

$$
\begin{aligned}
\eta=\left|\frac{\Delta D}{D}\right| \times 100 \%= & \left|-\frac{\Delta r}{r}\right| \times 100 \% \\
& =\frac{|\Delta r|}{r} \times 100 \%
\end{aligned}
$$

This expression means that the correction ratio $\eta$ only has a direct relation to the change ratio of the curvature radius $r$ of the cornea after the operation, which denotes that, if the change ratio of the curvature radius $r$ can be calculated, the rectification value of the refractive power can be obtained.

To model the corneal radial keratotomy by finite element method (FEM), the corneal geometry must be simplified and the corneal characteristics must be specified and approximated according to the experimental study. 
Table 1 Corneal variables and their values

\begin{tabular}{llll}
\hline Average thickness of cornea (mm) & 0.620 & Incision number & $4,8,12$ \\
Inner radius (mm) & 6.580 & Incision depth (of the thickness \%) & $60,80,90,95$ \\
Outer radius (mm) & 7.200 & Radius of optical zone (mm) & $1.3,1.8,2.3$ \\
Young's modulus (MPa) & 1.800 & Intraocular pressure (mm Hg) & $15,20,25,30$
\end{tabular}

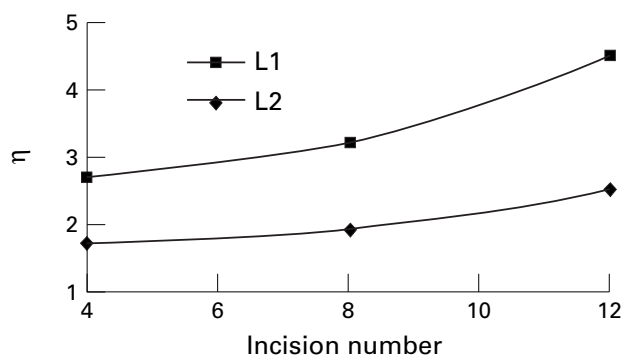

Figure 1 The effect of incision number on the change ratio of curvature radius $r$ after the operation. L1 shows a 95\% incision depth at intraocular pressure $25 \mathrm{~mm} \mathrm{Hg}$ and $\mathrm{L2}$ $60 \%$ incision at $20 \mathrm{~mm} \mathrm{Hg}$.

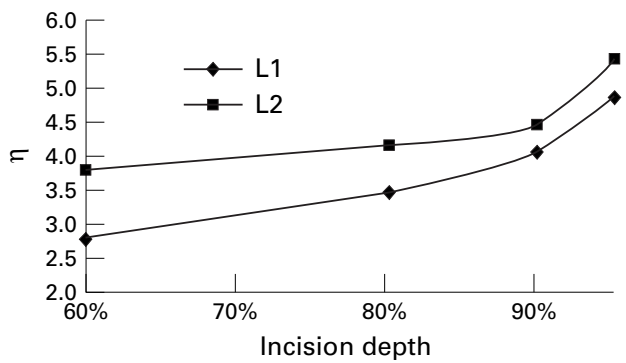

Figure 2 The effect of incision depth on the change ratio of the curvature radius $r$ after the operation. L1and L2 show the depth- $\eta$ relation at four incisions at intraocular pressure $20 \mathrm{~mm} \mathrm{Hg}$ and 12 incisions at $25 \mathrm{~mm} \mathrm{Hg}$, respectively.

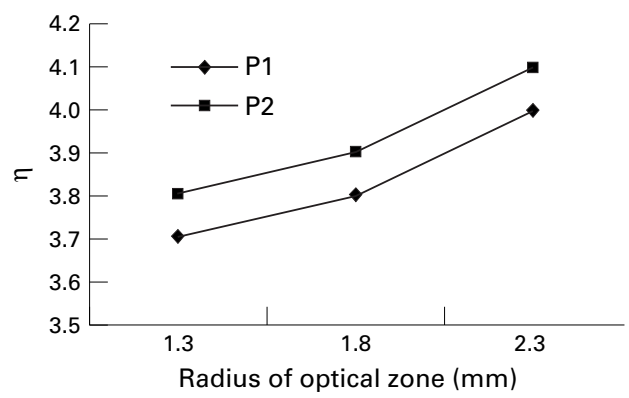

Figure 3 The effect of the optical zone radius on the change ratio of $r$ is positive. $P 1$ and $P 2$ are in two different intraocular pressure conditions.

This study is based on the following assumptions: (1) the cornea is elastic and non-linear; (2) it is isotropic; (3) it is basically uncompressible. Continuous media hypothesis and shell theory are the basis of the model and the computation..$^{5-7}$ Many important factors affecting the outcome of surgery have been taken into consideration and have even been optimised. The boundary condition and load type have been studied, a suitable set of which is applied to the mechanical models for computation. ${ }^{8}$

In order to study the effect of each of the variables on the operation outcome individually, a series of points are selected at the

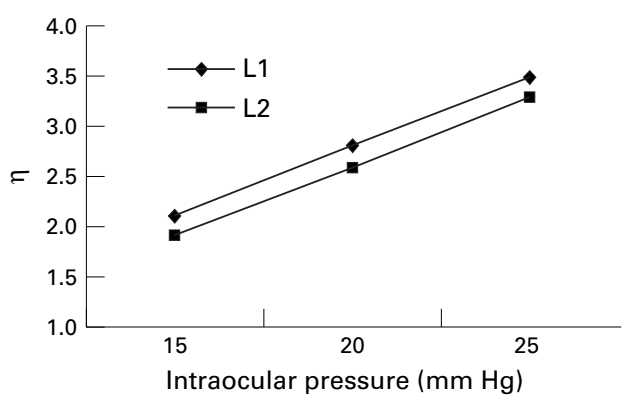

Figure 4 The effect of intraocular pressure on the change ratio of $r$ is positive. L1 is 12 incisions at $60 \%$ cut depth, and L2 eight incisions at $90 \%$ cut depth.

reasonable value of each variable, which is combined with that of other variables to construct a series of geometrical and biomechanical models of the cornea. The adopted variables of the cornea and the values of the variables selected are listed in Table 1 .

The computation work in this paper was done on the Sun working station with the help of ANSYs finite element analysis software (5.3 edition, USA Swanson Co Ltd).

\section{Results and conclusion}

Through the construction of the geometric and biomechanical models of the radial keratotomy operation, the simulated computation by FEM can give the different effects at the different operation variables.

After each model has been worked out, we can compare and analyse them on condition that one variable increasingly changes and the others remain constant. In order to reach a more comprehensive conclusion of the relation of the operational variables with change ratio of the curvature radius $r$, two models of other different conditions in the same controlled condition have been studied and plotted in the same figure (Fig 1).

From Figures 1-4 we can learn that all the four variables have a positive effect on the operational outcome; the greater they are the more correction can be gained. Therefore, the operational variables can be optimised to get the best result in different condition and different patient requirements.

The refractory system of the cornea comprises nearly $82 \%$ of the total refractory requirement of the eye's optical system. The other $18 \%$ is provided by other organs such as lens. The eye is a biological organ, not a pure optical device; hence, in constructing the models and computing, its physiological features should be noted.

We gratefully acknowledge the National Natural Science Foundation of China for the research grant that made this work possible.

1 George O, Waring I. Refractive keratotomy for myopia. Verlag 1985:911-17.

2 Hjortdal. Regional elastic performance of the human cornea. F Biomechanics 1996;29:931-4.

3 Maguire LJ, Singer DE, Klyce SD. Graphic presentation of Maguire LJ, Singer DE, Klyce SD. Graphic presentation of
computer-analyzed keratoscope photographs. Arch Ophthalmol 1987;105:223-30.

4 Liu Lin. Mordern refractive operation. People's Military Doctors'Press of China 1995:5-35. 
5 Yan-jun Zeng, Qing-hua Ren, Xiu-yun Li. Biomechanical properties of pig cornea. Acta Biophysica Sinica 1993;9: $323-7$

6 Yan-jun Zeng, Qing-hua Ren. Constitutive equation and stress relaxation of pig corneas. Chinese fournal of Biomedical Engineering 1995;14:360-4
7 Hoeltzel DA, Altman P, Buzard K, et al. Strip extensiometry for comparison of the mechanical response of bovine, rab8 Cazzani A, Atluri SN. Four-noded mixed finite elements Cazzani A, Atluri SN. Four-noded mixed finite elements
using unsymmetric stresses for linear analysis of membranes. Computational Mechanics 1993;114:229-51. 